\title{
RASSF2, a potential tumour suppressor, is silenced by CpG island hypermethylation in gastric cancer
}

\author{
M Endoh', G Tamura*,', T Honda', N Homma', M Terashima ${ }^{2}$, S Nishizuka ${ }^{3}$ and T Motoyama' \\ 'Department of Pathology, Yamagata University School of Medicine, 2-2-2 lida-nishi, Yamagata 990-9585, Japan; ²Department of Surgery, Fukushima \\ Medical University, I Hikarigaoka, Fukushima 960-1295, Japan; ${ }^{3}$ Molecular Therapeutics Program, Center for Cancer Research, National Cancer \\ Institute, National Institutes of Health, Bethesda, MD 20892, USA
}

\begin{abstract}
RASSF2, a member of the RASSFI family, has recently been identified as a potential tumour suppressor. We examined methylation status in multiple regions which included the CPG island and spanned the transcription start site of RASSF2 in 10 gastric cancer cell lines, as well as 78 primary gastric cancers and corresponding non-neoplastic gastric epithelia. Hypermethylation of RASSF2 in at least one of the regions examined was detected in seven (70\%) of the 10 cell lines; two (20\%) exhibited hypermethylation in all the regions examined including the transcription start site and lost expression of RASSF2 mRNA, which could, however, be restored by 5 aza- $2^{\prime}$ deoxycytidine treatment, while the other five (50\%) cell lines exhibited hypermethylation at the $5^{\prime}$ - and/or $3^{\prime}$ - edge, with four of them expressing RASSF2 mRNA. In primary gastric cancers and corresponding non-neoplastic gastric epithelia, frequencies of RASSF2 methylation ranged from 29\% (23 out of 78) to 79\% (62 out of 78) and 3\% (two out of 78) to 60\% (47 out of 78), respectively, at different CpG sites examined. Methylation was frequently observed at the $5^{\prime}$ - and $3^{\prime}$ - edges, and became less frequent near the transcription start site in both the primary gastric cancers and corresponding non-neoplastic gastric epithelia. Hypermethylation near the transcription start site was mostly cancer-specific. We thus showed that RASSF2 is silenced by hypermethylation near the transcription start site in gastric cancer. Hypermethylation was found initially to occur at the 5'- and 3'furthest regions of the $\mathrm{CpG}$ island in non-neoplastic gastric epithelia, to gradually spreads near the transcription start site to shut down RASSF2 expression, and ultimately to constitute a field-defect placing tissue increased risk for development of gastric cancer. British Journal of Cancer (2005) 93, I395-1399. doi:I0.1038/sj.bjc.6602854 www.bjcancer.com
\end{abstract}

Published online I November 2005

(c) 2005 Cancer Research UK

Keywords: RASSF2; hypermethylation; gastric cancer

Human cancers develop and progress by accumulation of both genetic and epigenetic alterations. Mutations of $p 53$ are frequent genetic (structural) alterations in gastric cancer (Tamura, 2002). Ecadherin is inactivated through any combinations of gene mutation, loss of heterozygosity ( $\mathrm{LOH})$, and promoter hypermethylation in gastric cancer, especially that of undifferentiated histological type (Tamura et al, 2001). Recent evidences of frequent silencing of $h M L H 1, p 16, R U N X 3$, and other tumour suppressor and tumour-related genes by promoter hypermethylation suggests that epigenetic alterations play the most important role in gastric carcinogenesis (Tamura, 2004). Furthermore, promoter hypermethylation initially occurs in non-neoplastic gastric epithelia, increases with age, and ultimately silences gene function to constitute a field-defect that may predispose tissues to development of gastric cancer (Waki et al, 2002, 2003b). Many genes become methylated in gastric epithelia during aging (Waki et al, 2003a), although frequencies of methylation depends on the sites of CpGs examined within a gene promoter (Satoh et al, 2002).

\footnotetext{
*Correspondence: Dr G Tamura;

E-mail: gtamura@med.id.yamagata-u.ac.jp

Received 12 April 2005; revised 3 October 2005; accepted 5 October 2005; published online I November 2005
}

Ras family genes are associated with the signal transduction from G-protein-coupled receptors and activation of the ras-signal transduction pathway frequently observed in human tumours (Shih et al, 1982; Malumbres and Pellicer, 1998; Ayllon and Rebollo, 2000). Ras proteins interact with a wide spectrum of regulators and downstream effectors to produce various cellular responses, including cell proliferation, differentiation, and apoptosis (Malumbres and Pellicer, 1998). Recently, the ras effectors/tumour suppressors RASSF1 and NORE1 were found to be inactivated by promoter hypermethylation in a variety of human tumours (Vavvas et al, 1998; Dammann et al, 2000; Pfeifer et al, 2002; Hesson et al, 2003; Vos et al, 2003b). RASSF2 was identified as a third member of the RASSF1 family and a ras effector/tumour suppressor (Vos et al, 2003a). RASSF2 binds to K-ras in a GPT-dependent manner via the Ras effector domain, but interacts with H-ras weakly (Vos et al, 2003a). RASSF2 inhibits the growth of tumour cells, promotes both cell cycle arrest and apoptosis, and is frequently downregulated in lung tumour cell lines (Vos et al, 2003a). The RASSF2-methylated RKO, colorectal cell line, without RASSF2 RNA expression showed increase of apoptosis and cell growth inhibition (Akino et al, 2005). These observations indicate that RASSF2 shows tumour suppressor activity in various human cancers.

In the present study, we examined the methylation status of RASSF2 at multiple regions which included the CpG island and 
spanned the transcription start site in gastric cancer cell lines, as well as primary gastric cancers and corresponding non-neoplastic gastric epithelia. The regions critical for methylation silencing of RASSF2 mRNA expression were examined by analyses of cell lines, while methylation spreading near the RASSF2 transcription start site was examined by analyses of primary gastric cancers and corresponding non-neoplastic gastric epithelia.

\section{MATERIALS AND METHODS}

\section{Samples}

A total of 10 gastric cancer cell lines with various histologies were cultured under appropriate conditions in our laboratory: MKN1, an adenosquamous cell carcinoma; MKN7, a well-differentiated adenocarcinoma; MKN28 and MKN74, moderately differentiated adenocarcinomas; MKN45 and KWS-I, poorly differentiated adenocarcinomas; KATO-III, a signet ring cell carcinoma; ECC10 and ECC12, endocrine cell carcinomas; and TSG 11, a hepatoid carcinoma. In total, 78 gastric cancer samples and matching nonneoplastic gastric tissues were obtained at surgery from 78 patients. Clinicopathological data were available for 71 of the 78 patients (Table 1). The patients ranged in age from 43 to 89 years (mean, 67.6 years). All the patients received follow-up care, for a median of 36.1 months (range, 1-77 months.). All samples were stored at $-80^{\circ} \mathrm{C}$ until processed. DNA was extracted from the 10 gastric cancer cell lines, 78 primary gastric cancers and corresponding non-neoplastic gastric epithelia using SepaGene

Table I Correlation between RASSF2 CpG island hypermethylation and clinicopathological characteristics in gastric cancer

\begin{tabular}{|c|c|c|c|}
\hline \multirow[b]{2}{*}{ Characteristics } & \multicolumn{2}{|c|}{ RASSF2 methylation status } & \multirow[b]{2}{*}{$P$-value } \\
\hline & Methylated at U I and DI & Others & \\
\hline Number of patients & 21 & 50 & \\
\hline Age (mean) & 69.4 & 66.9 & NS \\
\hline Gender & & & NS \\
\hline$M$ & 15 & 36 & \\
\hline $\mathrm{F}$ & 6 & 14 & \\
\hline Stage & & & NS \\
\hline Early & 7 & 8 & \\
\hline Advanced & 14 & 42 & \\
\hline Histological differentiation & & & NS \\
\hline Differentiated & 12 & 27 & \\
\hline Undifferentiated & 9 & 23 & \\
\hline Location & & & NS \\
\hline Upper & 4 & 9 & \\
\hline Middle & 6 & 18 & \\
\hline Lower & 10 & 19 & \\
\hline Unknown & । & 4 & \\
\hline Lymph node metastasis & & & NS \\
\hline Present & 11 & 31 & \\
\hline Absent & 10 & 19 & \\
\hline Lymphatic permeation & & & 0.033 \\
\hline ly $(-)$ & 7 & 6 & \\
\hline ly $(+)$ & 14 & 44 & \\
\hline Venous permeation & & & NS \\
\hline$\vee(-)$ & 8 & 10 & \\
\hline$v(+)$ & 13 & 40 & \\
\hline
\end{tabular}

$\mathrm{NS}=$ not significant by Fisher's exact probability test and Mann-Whitney's U-test.
(Sankyo-Junyaku, Tokyo, Japan). Total RNA was isolated from the 10 gastric caner cell lines with the TRIZOL reagent (Gibco BRL, Life Technologies, Gaithersburg, MD, USA).

\section{Methylation-specific PCR (MSP)}

Treatment of DNA samples with sodium bisulphite converts all unmethylated cytosines to uracils but does not affect methylated cytosines. Briefly, $2 \mu \mathrm{g}$ of genomic DNA was denatured by treatment with $\mathrm{NaOH}$ and modified by sodium bisulphite. The samples were then purified using Wizard DNA purification resin (Promega, Madison, WI, USA), treated with $\mathrm{NaOH}$, recovered in ethanol and resuspended in $30 \mu \mathrm{l}$ of distilled water. Amplification was performed in a $20 \mu \mathrm{l}$ reaction volume containing $2 \mu \mathrm{l}$ of GeneAmp PCR Gold Buffer (PE Applied Biosystems, Foster City, CA, USA), $1.0 \mathrm{~mm} \mathrm{MgCl}_{2}, 1 \mu \mathrm{l}$ of each primer, $0.2 \mu \mathrm{M}$ dNTPs, and $1 \mathrm{U}$ of Taq polymerase (AmpliTaq Gold DNA Polymerase, PE Applied Biosystems). Hot start PCR was performed in a thermal cycler (GeneAmp 2400, PE Applied Biosystems) for 35 cycles, each of which consisted of denaturation at $95^{\circ} \mathrm{C}$ for $15 \mathrm{~s}$, annealing at $55^{\circ} \mathrm{C}$ for $15 \mathrm{~s}$, and extension at $72^{\circ} \mathrm{C}$ for $30 \mathrm{~s}$, followed by a final 7min extension at $72^{\circ} \mathrm{C}$. A positive control and a negative control (distilled water without DNA) were included for each amplification. The PCR products were separated on a $6 \%$ nondenaturing polyacrylamide gel. To detect regions playing critical roles in regulating the expression of RASSF $2 A$ mRNA, we designed six primer sets spanning the RASSF2 transcription start site and including the $\mathrm{CpG}$ island (GenBank accession number AL 133354) (Figure 1). The following primer sets were used: $5^{\prime}$-GGT TTA AGT TTT TCG GTT TAT TC- $3^{\prime}$ and $5^{\prime}$-CAC GTC TAA CCG ACC CGC CAA ATC G-3' for the methylated RASSF2-U2 sequence (upstream 2; the upstream sequence furthest from the transcription start site) (212 bp); 5'-GGT TTA AGT TTT TTG GTT TAT TTG GA-3' ${ }^{\prime}$ and 5'-TCA CAT CTA ACC AAC CCA CCA AAT CA-3' for the unmethylated RASSF2-U2 sequence $(213 \mathrm{bp}) ; 5^{\prime}$-GTT TTT ATC GGA TTT GTT CGT TC- $3^{\prime}$ and $5^{\prime}$-CCA ACC CGA AAA AAT CGC TAA CGA CG- $3^{\prime}$ for the methylated RASSF2-U1 sequence (upstream 1; the upstream sequence nearest the transcription start site) (237 bp); $5^{\prime}$-GTT TTT ATT GGA TTT GTT TGT TTG-3' ${ }^{\prime}$ and 5'-CCA ACC CAA AAA AAT CAC TAA CAA CA-3' for the unmethylated RASSF2-U1 sequence $(237 \mathrm{bp})$; $5^{\prime}$-GTA TTT CGC GTT AGT GTT TC-3' and $5^{\prime}$-TTA AAC CCG ACC CGC CGA TCG$3^{\prime}$ for the methylated RASSF2-D1 sequence (downstream 1; the downstream sequence nearest the transcription start site in $\mathrm{CpG}$ island) (183 bp); $5^{\prime}$-TTG GGT ATT TTG TGT TAG TGT TTT GTT$3^{\prime}$ and $5^{\prime}$-ATT TAA ACC CAA CCC ACC AAT CAA T- $3^{\prime}$ for the unmethylated RASSF2-D1 sequence (189 bp); $5^{\prime}$-CGG GTT TAA AAA GAA GGA AGG AC- $3^{\prime}$ and $5^{\prime}$-GCG CGA ACC CCC GCC AAA AAC CG-3 $3^{\prime}$ for the methylated RASSF2-D2 sequence (135 bp); $5^{\prime}$-GTG GGT TTA AAA AGA AGG AAG GAT-3' and 5'-CAC ACA AAC CCC CAC CAA AAA CCA T- $3^{\prime}$ for the unmethylated RASSF2D2 sequence (137 bp); 5'-TTC GTT TAG AAG ACG GCG GC-3' and $5^{\prime}$-CCT TCC TTC TTT TTA AAC CCG- $3^{\prime}$ for the methylated

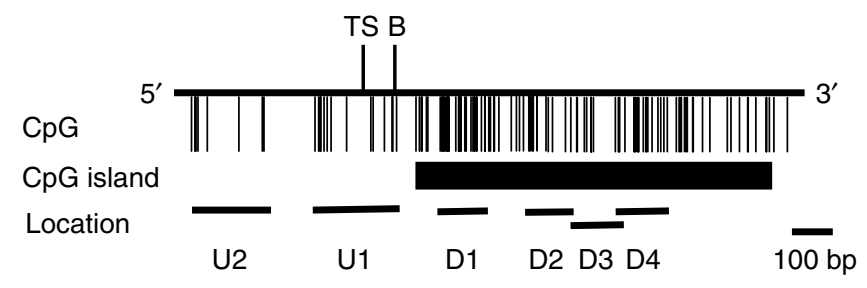

Figure I Location of methylation-specific PCR (MSP) near the transcription start site including (A) CPG island of RASSF2. Vertical lines indicate individual $\mathrm{CpG}$ sites. TS, transcription start site; (B) exon I and intron I boundary. 
RASSF2-D3 sequence ( $166 \mathrm{bp}) ; 5^{\prime}$-AAT TTG TTT AGA AGA TGG TGG T- $3^{\prime}$ and $5^{\prime}$-ATC CTT CCT TCT TTT TAA ACC CA- $3^{\prime}$ for the unmethylated RASSF2-D3 sequence $(170 \mathrm{bp}) ; 5^{\prime}$-CGA AGG AGG GCG GGG AGA TC- $3^{\prime}$ and $5^{\prime}$-TCC GCC GCC GTC TTC TAA ACG-3' for the methylated RASSF2-D4 sequence (downstream 4; the downstream sequence of furthest from the transcription start site) (148 bp); and 5'-GTT TTG AAG GAG GGT GGG GAG ATT-3' and $5^{\prime}$-AAT CCA CCA CCA TCT TCT AAA CA- $3^{\prime}$ for the unmethylated RASSF2-D4 sequence $(154 \mathrm{bp})$. The positions of amplified regions are shown in Figure 1. As a positive control, Sss I methylase (New England BioLabs, Inc., Beverly, MA, USA) was used to methylate $100 \mathrm{mg}$ of liver tissue-derived DNA obtained from an autopsy sample, and was modified by sodium bisulphite as described above.

\section{Reverse transcription - PCR (RT - PCR)}

Isolated RNA was reverse-transcribed and amplified using a onestep RT-PCR System (Gibco BRL). Primer sequences used were $5^{\prime}$-AAG ACA TCC GTG TTC ACA CC-3' and 5'-TCG TTC TCA TGG CTC AGA TT- $3^{\prime}$ for RASSF2A mRNA (462 bp); and sense, $5^{\prime}$-AAA TCT GGC ACC ACA CCT T- $3^{\prime}$ and antisense, $5^{\prime}$-AGC ACT GTG TTG GCG TAC AG-3' for $\beta$-actin $(646 \mathrm{bp})$. Primers for RASSF $2 A$ mRNA can also amplify RASSF $2 B$ mRNA product, but product size of RASSF $2 B$ mRNA ( $409 \mathrm{bp}$ ) is smaller than that of RASSF2A mRNA. Thus, they could be differentiable.

\section{5-aza-2'-deoxycytidine (5-aza-dC) treatment}

To examine restoration of RASSF2 mRNA expression, two cell lines, KATO-III and KWS-I, were incubated for $96 \mathrm{~h}$ with $5 \mu \mathrm{M}$ 5-aza-dC (Sigma, St Louis, MO, USA), and then harvested for RNA extraction and RT-PCR. MKN74, which expresses RASSF2 mRNA with an unmethylated CpG island, was used as a control.

\section{Statistical analysis}

Statistical comparisons were performed using Fisher's exact probability test and Mann-Whitney's $U$-test. Values of $P<0.05$ were considered significant.

\section{RESULTS}

Methylation status and mRNA expression of RASSF2 in gastric cancer cell lines

Hypermethylation of RASSF2 in at least one of the regions examined was detected in seven of the 10 cell lines, MKN1, MKN7, MKN28, MKN45, KATO-III, KWS-I, and ECC10 (Figure 2A). Among these, KATO-III and KWS-I exhibited hypermethylation in all the regions examined, and lost RASSF2 mRNA expression (Figure $2 \mathrm{~A}$ ), which, however, was restored by 5 -aza-dC treatment (Figure 2B). The other five of these seven cell lines, MKN1, MKN7, MKN28, MKN45, and ECC10 exhibited methylation only in outskirt regions, and four of them expressed RASSF2 mRNA (Figure 2A). Three cell lines, MKN74, TSG11, and ECC12, did not exhibit hypermethylation in any region. TSG11 and ECC12 lost RASSF2 mRNA expression despite being unmethylated.

Hypermethylation RASSF2 CpG island in primary gastric cancer and corresponding non-neoplastic gastric epithelia

Methylation frequencies of $R A S S F 2$ varied in the regions upstream and downstream of the transcription start site. Of primary gastric cancers and corresponding non-neoplastic epithelia, hypermethylation was observed in $55 \%$ (43 out of 78 ) and $36 \%$ ( 28 out of 78 ) at $\mathrm{U} 2,29 \%$ (23 out of 78 ) and 3\% (two out of 78 ) at U1, $42 \%$ (33 out

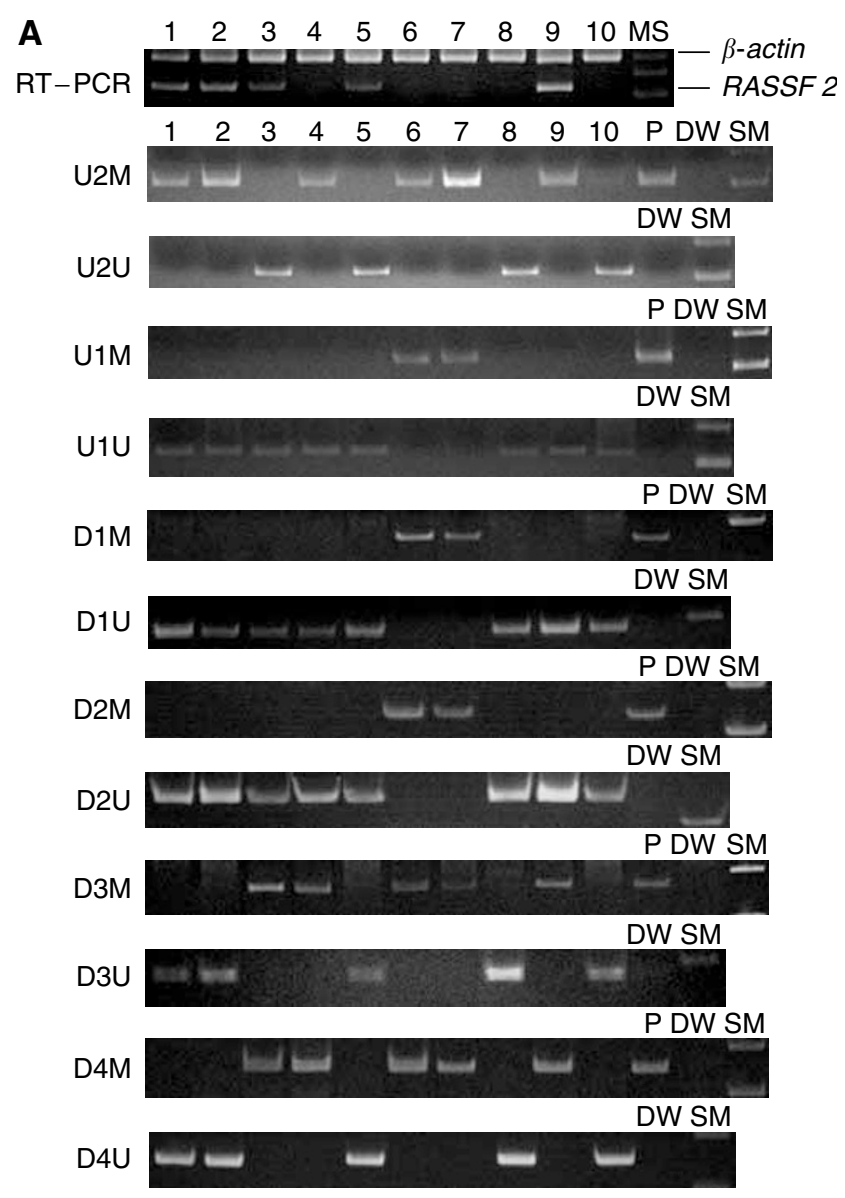

B

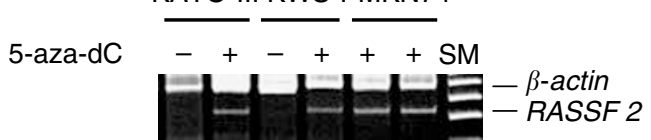

Figure 2 Reverse transcription-PCR and methylation-specific PCR (MSP) (A), and comparison of RASSF2 mRNA expression before (-) and after $(+)$ 5-aza-dC treatment $(\mathbf{B})$ in gastric cancer cell lines. (A) RASSF2 mRNA is present in lanes I $-3,5$, and 9. Methylated-PCR products from $U 2 M$ to D4M are present at all the regions in lanes 6 and 7 , at only the $5^{\prime}$ - and/or 3'- edge in lanes $1-4$, and 9, and not present in lanes 5, 8, and 10. Unmethylated-PCR products from $U 2 \cup$ to D4U are alternatively present in lanes $1-5$, and $8-10$, and not present in lanes 6 and 7 . Lanes I, MKNI; 2, MKN7; 3, MKKN28; 4, MKN45; 5, MKN74; 6, KATO-III; 7, KWS-I; 8, TSGII; 9, ECCI0; 10, ECC I2; P, positive control; DW, distilled water; and SM, size marker. (B) Treatment with 5-aza-dC restored RASSF2 mRNA expression in KATO-III and KWS-l, but did not affect levels of RASSF2 expression in MKN74.

of 78 ) and $6 \%$ (five out of 78) at D1, $45 \%$ (35 out of 78 ) and $13 \%$ (10 out of 78 ) at D2, $54 \%$ (42 out of 78 ) and $28 \%$ (22 out of 78 ) at D3, 79\% (62 out of 78 ) and $60 \%$ (47 out of 78) at D4, respectively (Figures 3 and 4). Unmethylated RASSF2 sequence was present in all the samples (data not shown). Gastric cancers with methylation at U1 and D1 exhibited significantly less frequent lymphatic permeation than unmethylated gastric cancers (Table 1) No significant correlation was observed between methylation status of RASSF2 and other clinicopathological factors. Methylation status of RASSF2 did not significantly influence eventfree survival rate, as determined by Kaplan-Meier curve analysis with the log-rank test and Breslow-Gehan-Wilcoxon test (data not shown). 


\section{DISCUSSION}

Results in gastric cancer cell lines suggest that hypermethylation near the transcription start site was associated with loss of RASSF2 mRNA expression, although other mechanisms might also contribute to RASSF2 silencing. While by analyses of samples from primary gastric cancer patients, frequencies of methylation at each region investigated were higher in primary gastric cancers than in corresponding non-neoplastic gastric epithelia. Very recently, Akino et al reported the significant association between methylation and expression status of RASSF2 in colon cancer cell lines and primary colorectal cancers. They identified the RASSF2 promoter region and showed that absence of RASSF2 transcription was caused by DNA methylation, not by alteration

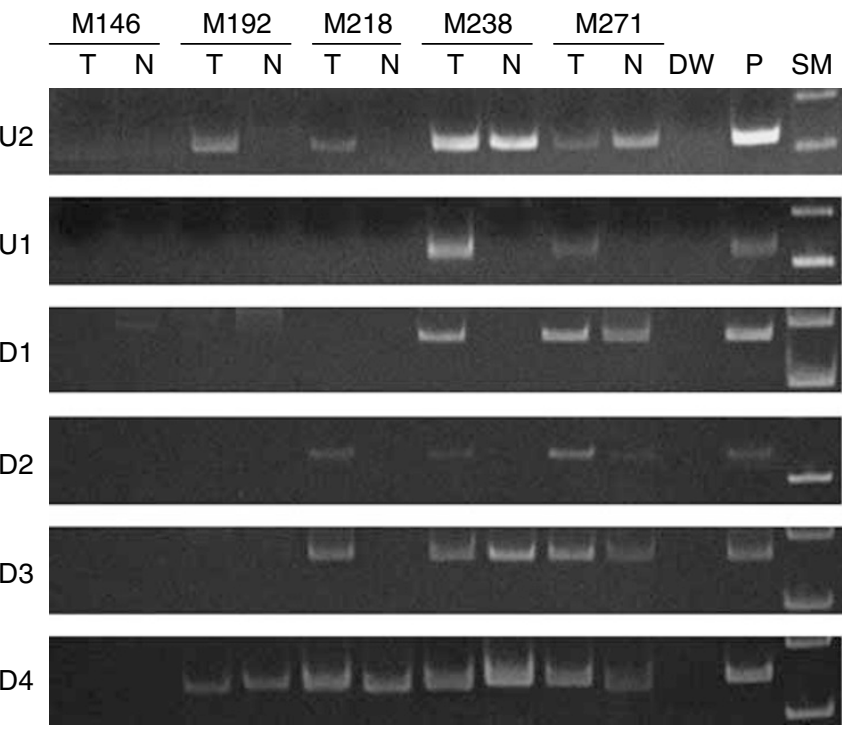

Figure 3 Examples of methylation-specific PCR (MSP) in primary gastric cancers $(T)$ and corresponding non-neoplastic gastric epithelia $(N)$. Methylation is frequent in outskirt regions (U2 and D4), but becomes less frequent near the transcription start site $(U I$ and $D I)$ in both gastric cancers and non-neoplastic gastric epithelia. DW, distilled water; P, positive control; and SM, size marker. of transcription factors, and also showed histone acetylation at the $5^{\prime}$ region of $R A S S F 2$ in colorectal cancer cell lines with DNA methylation (Akino et al, 2005). It has also been reported that inactivation of RASSF2 with siRNA increased phospholilation of MAP kinase, and that RASSF2 suppressed downstream signalling in the RAS pathway (Akino et al, 2005). Colorectal tumours with RASSF2 methyaltion showed $K$-ras/BRAF mutations significantly more frequently than those without RASSF2 methyaltion (Akino et al, 2005), although earlier studies have showed that $K$-ras mutation and RASSF2 methylation are mutually exclusive (Hesson et al, 2005). In the present study, we have showed that RASSF2 methylation is frequent in gastric cancer, despite the rarity of $K$-ras/BRAF mutations in this tumour (Kim IJ et al, 2003).

Methylation in the $5^{\prime}$ - and $3^{\prime}$ - furthest regions within the CpG island was very frequent in both primary gastric cancers and corresponding non-neoplastic gastric epithelia, but became less frequent near the transcription start site. Methylation near the transcription start sites at U1 and D1 appeared to be mostly cancer-specific. These findings are essentially the same as those for DAP-kinase hypermethylation (Satoh et al, 2002). Therefore, the pattern of spread of methylation, initially at the outskirts of CpG islands and then progressing to regions critical for gene silencing, might be common to various types of methylation-related gene silencing. Since frequencies of methylation are influenced by the location of MSP, as noted above, the significance of detection of DNA methylation in a giver tumour suppressor or tumour-related gene varied significantly. For example, hypermethylation near the transcription start site, which is cancer-specific and results in gene silencing, can be used as a diagnostic marker of malignancy in tissues or other samples, such as serum or ascites, and hypermethylation at a region next to such a critical region might be an early signal of carcinogenesis.

Gastric cancers with methylation at $\mathrm{U} 1$ and D1, a change critical for RASSF2 silencing, exhibited significantly less frequent lymphatic permeation than unmethylated gastric cancers. Gastric cancers with a high frequency of microsatellite instability (MSI-H), which is the result of hMLH1 silencing by hypermethylation, exhibited less frequent lymph node metastasis (dos Santos et al, 1996; Wu et al, 2000), and several genes are simultaneously methylated together with $h M L H 1$ (Kim H et al, 2003; Homma et al, 2005).

In conclusion, hypermethylation of RASSF 2 initially occurs in the $5^{\prime}$ - and $3^{\prime}$ - outskirt regions of the CpG island in non-neoplastic

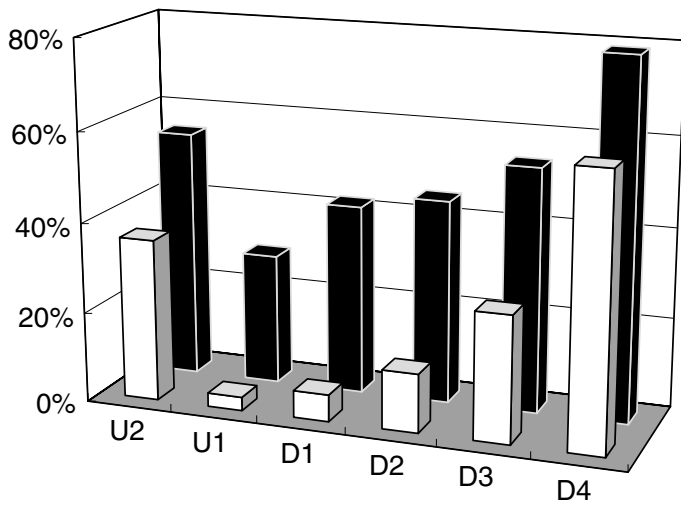

$\square$ Non-neoplastic gastric epithelia $(N=78)$

- Gastric cancer $(N=78)$

\begin{tabular}{lrrrrrr}
\hline & U2 & \multicolumn{1}{c}{ U1 } & \multicolumn{1}{c}{ D1 } & D2 & \multicolumn{1}{c}{ D3 } & D4 \\
\hline Gastric cancer $(N=78)$ & $55 \%(43)$ & $29 \%(23)$ & $42 \%(33)$ & $45 \%(35)$ & $54 \%(42)$ & $79 \%(62)$ \\
Non-neoplastic gastric epithelia $(N=78)$ & $36 \%(28)$ & $3 \%(2)$ & $6 \%(5)$ & $13 \%(10)$ & $28 \%(22)$ & $60 \%(47)$
\end{tabular}

Figure 4 Percentages of hypermethylation in various regions (U2-D4) of RASSF2. 
gastric epithelia, spreads near the transcription start site to shut down RASSF2 expression, and ultimately constitutes a field-defect which places tissue at increased risk for the development of gastric cancer.

\section{REFERENCES}

Akino K, Toyota M, Suzuki H, Mita H, Sasaki Y, Ohe-toyota M, Issa JP, Hinoda Y, Imai K, Tokino T (2005) The Ras effector RASSF2 is a novel tumor-suppressor gene in human colorectal cancer. Gastroenterology 129: $156-269$

Ayllon V, Rebollo A (2000) Ras-induced cellular events. Mol Membr Biol 17: $65-73$

Dammann R, Li C, Yoon JH, Chin PL, Bates S, Pfeifer GP (2000) Epigenetic inactivation of a RAS association domain family protein from the lung tumor suppressor locus 3p21.3. Nat Genet 25: 315-319

dos Santos NR, Seruca R, Constancia M, Seixas M, Sobrinho-Simoes M (1996) Microsatellite instability at multiple loci in gastric carcinoma: clinicopathologic implications and prognosis. Gastroenterology 110: $38-44$

Hesson L, Dallol A, Minna JD, Maher ER, Latif F (2003) NORE1A, a homologue of RASSF1A tumor suppressor gene is inactivated in human cancers. Oncogene 22: $947-954$

Hesson L, Wilson R, Morton D, Adams C, Walker M, Maher ER, Latif F (2005) CpG island promotor hypermethylation of a novel Ras-effector gene RASSF2A is an early in colon carcinogenesis and correlates inversely with K-ras mutation. Oncogene 24: 3987-3994

Homma N, Tamura G, Honda T, Jin Z, Ohmura K, Kawata S, Motoyama $\mathrm{T}$ (2005) Hypermethylation of Chfr and hMLH1 in gastric noninvasive and early invasive neoplasias. Virchows Arch 446: 120-126

Kim H, Kim YH, Kim SE, Kim NG, Noh SH, Kim H (2003) Concerted promoter hyper-methylation of hMLH1, p16INK4A, and E-cadherin in gastric carcinomas with microsatellite instability. J Pathol 200: $23-31$

Kim IJ, Park JH, Kang HC, Shin Y, Park HW, Park HR, Ku JL, Lim SB, Park JG (2003) Mutational analysis of BRAF and K-ras in gastric cancers: absence of BRAF mutations in gastric cancers. Hum Genet 114: 118-120

Malumbres M, Pellicer A (1998) RAS pathways to cell cycle control and cell transformation. Front Biosci 3: 887-912

Pfeifer GP, Yoon JH, Liu L, Tommasi S, Wilczynski SP, Dammann R (2002) Methylation of the RASSF1A gene in human cancers. Biol Chem 383: 907-914

Satoh A, Toyota M, Itoh F, Kikuchi T, Obata T, Sasaki Y, Suzuki H, Yawata A, Kusano M, Fujita M, Hosokawa M, Yanagihara K, Tokino T, Imai K (2002) DNA methylation and histone deacetylation associated with

\section{ACKNOWLEDGEMENTS}

This work was supported in part by a Grant-in-Aid for Cancer Research (15-20) from the Ministry of Health, Labor and Welfare of Japan.

silencing DAP kinase gene expression in colorectal and gastric cancers. Br J Cancer 86: 1817-1823

Shih TY, Stokes PE, Smythers GW, Dhar R, Oroszlan S (1982) Characterization of the phosphorylation sites and the surrounding amino acid sequences of the $\mathrm{p} 21$ transforming proteins coded for by the Harvey and Kirsten strains of murine sarcoma viruses. J Biol Chem 257: $11767-11773$

Tamura G (2002) Genetic and epigenetic alterations of tumor suppressor and tumor-related genes in gastric cancer. Histol Histopathol 17: $323-329$

Tamura G (2004) Promoter methylation status of tumor suppressor and tumor-related genes in neoplastic and non-neoplastic gastric epithelia. Histol Histopathol 19: $221-228$

Tamura G, Sato K, Akiyama S, Tsuchiya T, Endoh Y, Usuba O, Kimura W, Nishizuka S, Motoyama T (2001) Molecular characterization of undifferentiated-type gastric carcinoma. Lab Invest 81: 593-598

Vavvas D, Li X, Avruch J, Zhang XF (1998) Identification of Norel as a potential Ras effector. J Biol Chem 273: 5439-5442

Vos MD, Ellis CA, Elam C, Ulku AS, Taylor BJ, Clark GJ (2003a) RASSF2 is a novel K-Ras-specific effector and potential tumor suppressor. J Biol Chem 278: 28045-28051

Vos MD, Martinez A, Ellis CA, Vallecorsa T, Clark GJ (2003b) The proapoptotic Ras effector Nore1 may serve as a Ras-regulated tumor suppressor in the lung. J Biol Chem 278: 21938-21943

Waki T, Tamura G, Sato M, Motoyama T (2003a) Age-related methylation of tumor suppressor and tumor-related genes: an analysis of autopsy samples. Oncogene 22: $4128-4133$

Waki T, Tamura G, Sato M, Terashima M, Nishizuka S, Motoyama T (2003b) Promoter methylation status of DAP-kinase and RUNX3 genes in neoplastic and non-neoplastic gastric epithelia. Cancer Sci 94: $360-364$

Waki T, Tamura G, Tsuchiya T, Sato K, Nishizuka S, Motoyama T (2002) Promoter methylation status of E-cadherin, hMLH1, and p16 genes in nonneoplastic gastric epithelia. Am J Pathol 161: 399-403

Wu MS, Lee CW, Shun CT, Wang HP, Lee WJ, Chang MC, Sheu JC, Lin JT (2000) Distinct clinicopathologic and genetic profiles in sporadic gastric cancer with different mutator phenotypes. Genes Chromosomes Cancer 27: $403-411$ 